\title{
Histologic and immunohistochemical decision-making in endometrial adenocarcinoma
}

\author{
Lesley Lomo ${ }^{1}$, Marisa R Nucci ${ }^{1}$, Kenneth R Lee ${ }^{1}$, Ming-Chieh Lin $^{2}$, Michelle S Hirsch ${ }^{1}$, \\ Christopher P Crum ${ }^{1}$ and George L Mutter ${ }^{1}$ \\ ${ }^{1}$ Division of Women's and Perinatal Pathology, Department of Pathology, Brigham and Women's Hospital, \\ Boston, MA, USA and ${ }^{2}$ Department of Pathology, National Taiwan University Hospital, Taipei, Taiwan
}

\begin{abstract}
Diffuse p53 immunostaining distinguishes $85 \%$ of serous (Type II) from endometrioid (Type I) carcinomas and is an independent marker for poor prognosis. Interobserver reproducibility for the diagnosis of these entities, as well as selection and prediction of p53 immunostaining results, is unknown. Reproducibility of three pathologists regarding: (1) a two (I and II) and (2) three part classification (I, II or indeterminate); (3) recommendation for p53 staining and (4) expectations of p53 staining results were computed with the kappa $(k)$ statistic. All cases were immunostained for p53 and independently scored. A two and three tiered classification scheme achieved high $(k=0.71)$ and moderate $(k=0.49)$ reproducibility. Non-unanimous cases were more likely to be reclassified into an 'indeterminate' category ( 27 cases, $39 \%$ of passes) compared to those with unanimous ( 82 cases, $14 \%$ of passes) classification. Pathologists recommended p53 immunostaining with poor $(k=0.28)$ reproducibility, but staining prediction was made with good concordance $(69 \%, k=0.50)$. Moreover, p53 staining was more common in diagnostically discordant $(46 \%)$ compared to concordant (16\%) cases. A subset of endometrial cancers do not readily fit within a two-class system and can be culled from cases that (1) do not achieve interobserver concordance and (2) are more likely to be chosen for p53 immunostaining and (3) are more likely to stain positive for p53. Because p53 is an important marker for endometrial adenocarcinoma outcome, and cannot be predicted in advance in indeterminate cases, p53 immunostaining should be employed in cases with observer disagreement in a binary system.

Modern Pathology (2008) 21, 937-942; doi:10.1038/modpathol.2008.97; published online 23 May 2008
\end{abstract}

Keywords: p53; endometrial neoplasms; serous carcinoma; reproducibility

Endometrial adenocarcinoma is a biologically diverse disease that has been divided into two subgroups based on histomorphology, biologic behavior, and underlying genetic aberrations. Type I tumors exhibit endometrioid morphology, arise in the setting of increased estrogen exposure with subsequent development of precancerous lesions (endometrial intraepithelial hyperplasia-EIN/atypical hyperplasia), are associated with frequent PTEN inactivation mutations, and have a favorable outcome. $^{1,2}$ By contrast, Type II tumors demonstrate papillary serous or clear cell morphology, are not associated with any known precursor lesions, demonstrate frequent p53 mutations, and are asso-

Correspondence: Dr CP Crum, MD, Department of Pathology, Brigham and Women's Hospital, 75 Francis Street, Boston, MA 02115, USA.

E-mail: ccrum@partners.org

Received 31 March 2008; revised 25 April 2008; accepted 26 April 2008; published online 23 May 2008 ciated with a poor outcome. ${ }^{3,4}$ The differences between these groups are summarized in Table 1. The observation that uterine papillary serous carcinomas have a greater tendency to metastasis and/ or recurrence in the peritoneum underscores the importance of accurate diagnosis. ${ }^{5}$

Although the distinction between serous and endometrioid subgroups of endometrial cancer has a strong theoretical basis, subsets of endometrial adenocarcinoma have been recognized that do not fall cleanly into either of the two categories. Tumors with glandular architecture that more closely approximate endometrioid adenocarcinoma may occasionally demonstrate strong p53 positivity. ${ }^{6,7}$ Alternatively, mixed pattern tumors exhibiting coexisting but immunophenotypically distinct serous and endometrioid patterns have also been encountered. Finally, recent reports have identified putative precursor lesions (endometrial glandular dysplasia) that are associated with uterine papillary serous carcinoma but are distinct from serous 
Table 1 Pathogenetic subsets of endometrial carcinoma

\begin{tabular}{|c|c|c|}
\hline Parameter & Type I & Tyре II \\
\hline Histology & Endometrioid & $\begin{array}{l}\text { Papillary serous or } \\
\text { mixed }\end{array}$ \\
\hline Age & $50 s-60 s$ & $60 s-70 s$ \\
\hline Estrogenic stimuli & Common & Uncommon \\
\hline $\begin{array}{l}\text { Background } \\
\text { Endometrium }\end{array}$ & Anovulatory & Atrophic \\
\hline Precursor & EIN & Unknown \\
\hline Transition & Slow & Rapid \\
\hline Molecular genetics & $\begin{array}{l}\text { MSI, PTEN } \\
\text { mutation }\end{array}$ & $\begin{array}{l}\text { p53 mutations, } 1 p \\
\text { deletions }\end{array}$ \\
\hline Familial & HNPCC & Not known \\
\hline Spread & Lymph nodes & Peritoneum \\
\hline Concurrent ovarian & Common & Uncommon \\
\hline Prognosis & Good & Poor \\
\hline
\end{tabular}

endometrial intraepithelial carcinoma. ${ }^{8,9}$ Owing to the marked differences in outcome associated with serous morphology or p53 inactivation, the accurate assignment of tumor type has important clinical implications, both in terms of therapy and prognosis. $^{10}$

The purpose of this study was to identify parameters that influence the accuracy and reproducibility of endometrial adenocarcinoma classification into prototypical Type I and II groups. Indications and expected results for p53 immunohistochemistry were compared for multiple observers with actual results. The ability of pathologists to consistently identify a mixed, or intermediate, class of lesions was directly measured, and the immunophenotype of this group examined.

\section{Materials and methods}

\section{Case Selection and Review}

In all 103 consecutive cases of endometrial adenocarcinoma were selected from the diagnostic files of Brigham and Women's Hospital for the period 19882002, including hysterectomies and endometrial biopsies. H\&E slides were examined by three blinded independent reviewers (KL, MN, and GM) who were asked to score all cases using two classification systems:

\section{Classification Systems}

A classic 'best fit' diagnostic schema for classification employed a binary system where Type I corresponded to endometrioid adenocarcinoma and Type II to papillary serous carcinoma.

An expanded 3-class schema provided for an uncertain category in addition to Type I (endometrioid) and Type II (serous) tumors. Observers were permitted to select an indeterminate category, corresponding to patterns that were not easily categorized into either of the classic groups.
All cases identified by at least one pathologist as 'Uncertain' in the expanded schema were then resolved by consensus review into subgroups. These subgroups included specimens which were indeterminate due to technical reasons, carcinomas with discrete admixed or hybrid histologies, and other problems in classification.

\section{Other Correlations}

In addition to classifying the tumors into the aforementioned groups, the observers recorded their preference for performing immunostaining for p53 and were asked to predict the p53 immunostaining pattern.

\section{Immunohistochemistry}

All cases were immunostained for p53, results of which were scored by two other pathologists blinded to the previous histologic interpretations:

P53-negative

This group had $<50 \%$ positive staining nuclei (Figure 1a).

\section{P53-positive}

This group required one of the following: (1) $50 \%$ or more nuclei staining positive (Figure 1b), or (2) Two discrete geographic patterns in which one stained with $50 \%$ or more positive nuclei (Figure 1c).

\section{Statistics}

Concordance in histologic diagnosis between observers or between observers and immunohistochemistry were assessed using the kappa statistic. Values of $k$ were interpreted as poor to fair (less than $0.4)$ good (0.4-0.55), very good (0.55-0.7) and excellent (greater than 0.7). The following correlations were made and are illustrated in the results in dendrograms signifying a hierarchical clustering algorithm using Ward's linkages in Euclidian space:

(1) Observer agreement in classifying endometrial adenocarcinoma under a two or three category system.

(2) Observer agreement in ordering p53 immunostaining and predicting p53 immunostaining results

(3) Relationship between a diagnosis of 'indeterminate', interobserver agreement and p53 immunostaining.

Each dendrogram depicts all of the cases individually as rows, and the interpretation of each reviewer in columns. When observers agree on a given interpretation for a specific case, the line color is continuous across all three columns. When only one or two observers agree, the line is interrupted by 

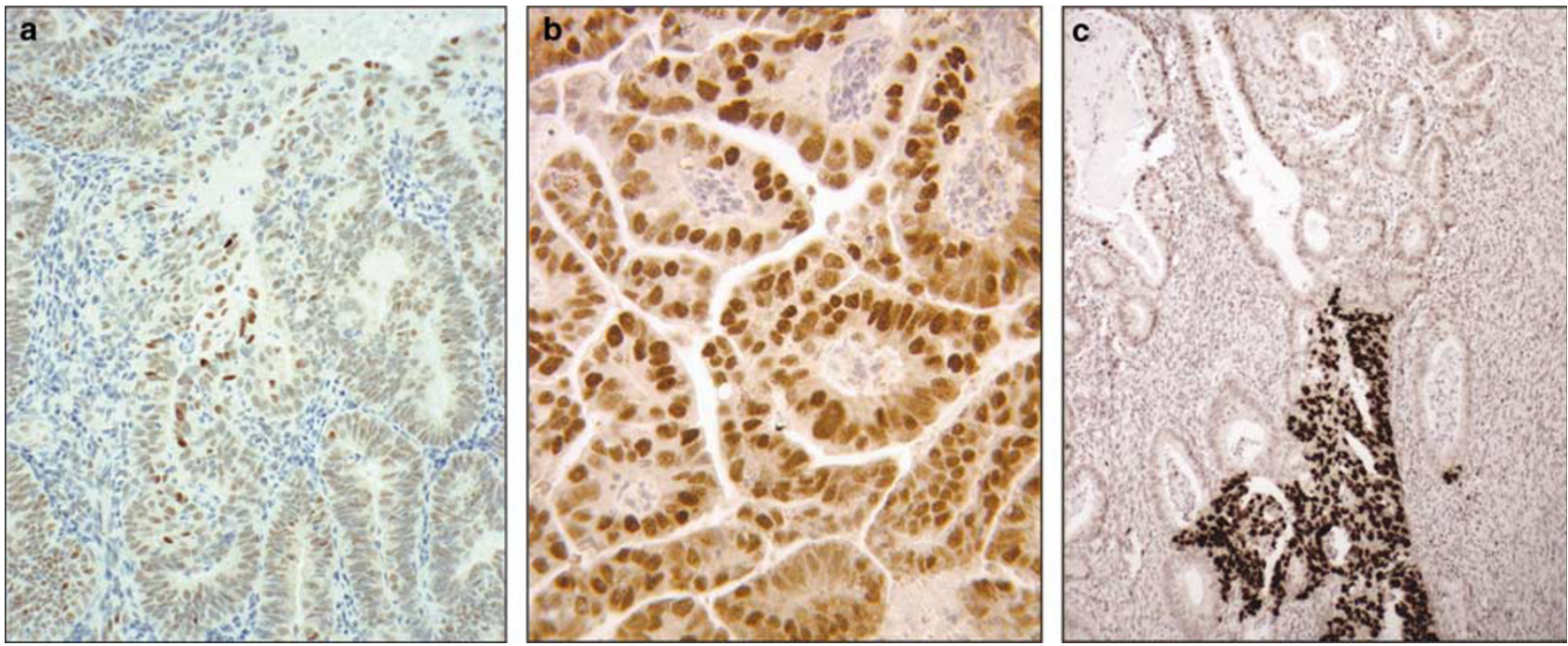

Figure 1 Patterns of immunostaining for p53. (a) Heterogeneous staining involving less than 50\% (typically 0-15\%) of tumor cell nuclei. (b) Homogeneous staining of over $50 \%$ of tumor cell nuclei. (c) Heterogeneous (upper) juxtaposed with homogeneous (lower) staining.

a different color in the column of the non-concordant observer for that case. In each analysis, cases are reordered to arrange those with unanimous, majority and minority agreement into clusters. The latter device provides a rapid visual assessment of the level of concordance across observers (Figures 2 and 3).

\section{Results}

\section{Interobserver Agreement of Lesion Classification}

Figure 2 displays cases with uniform agreement for Type I and II adenocarcinoma (Figures 2a and b) and non-uniform agreement (Figure 2c) under a binary classification system. Using a binary classification system, reviewers achieved very good to excellent interobserver reproducibility $(k=0.71$, Figure $2 d)$. All three observers agreed on the diagnosis of Type I or Type II in 84 and 72 percent of cases respectively (Table 2). Examples of cases unanimously classified as Type I and II are illustrated in Figure 2a and b.

When the expanded (3-group) classification system was used by the reviewers, concordance was good but the reduction in concordance relative to the binary system reflected variability in re-classification of cases as indeterminate (overall $k=0.49$, Figure 2e). Two or more observers agreed on the diagnosis of Type I or Type II in 78 and 83 per cent of cases (Table 2). Of the 44 cases receiving a score (by at least one observer) of 'indeterminate', two or more observers agreed on this diagnosis in 34\%. Examples of cases in this category are illustrated in Figures $3 a-d$.

\section{Classification and p53 Immunostaining}

There was poor interobserver concordance for the selection of p53 immunostaining, reflecting different thresholds of observer commitment to this test $(k=0.28$, Figure 2f). However, in terms of p53 staining outcome, the proportion of cases scoring positive for p53 closely paralleled the certainty of the diagnosis (Table 2, Figure 3). Moreover, the p53staining outcome paralleled the expectations of the observers, irrespective of whether the stain would have been ordered. When an expanded classification was used, only $3 \%$ of cases with a unanimous diagnosis of Type I scored p53-positive. This contrasts with $88 \%$ of cases with a unanimous diagnosis of Type II. Depending on the observer, 19 to 21 percent of cases were re-classified as indeterminate using the expanded system (Table 2) and from 50 to 80 per cent of these cases scored positive for p53 (Table 2). In 15 cases, at least two of three observers were concordant in assignment to the uncertain class. Examples of indeterminate cases scoring p53-positive are illustrated in Figure 3.

\section{Resolution of Cases Classified as Indeterminate}

Overall, in 44 cases, $1 / 3$ (29) $2 / 3$ (13) or $3 / 3$ (2) observers reclassified cases into a third category. The most common reasons were overlapping histologic characteristics. These are illustrated in Figure $3 a-d$ and the correlation of p53 staining with expectations is summarized in Figure 3e. Cases in the indeterminate category included those with discordances in cytohistologic architecture, in which an endometrioid growth pattern was accompanied by a higher than expected nuclear grade (Figure 3a and b), a perception of two discrete histologic patterns (Figure 3c) and villoglandular architecture that prompted a diagnosis of indeterminate (Figure 3d). Others included diagnostic uncertainty due to stated difficulties in classification, sampling and preservation. 

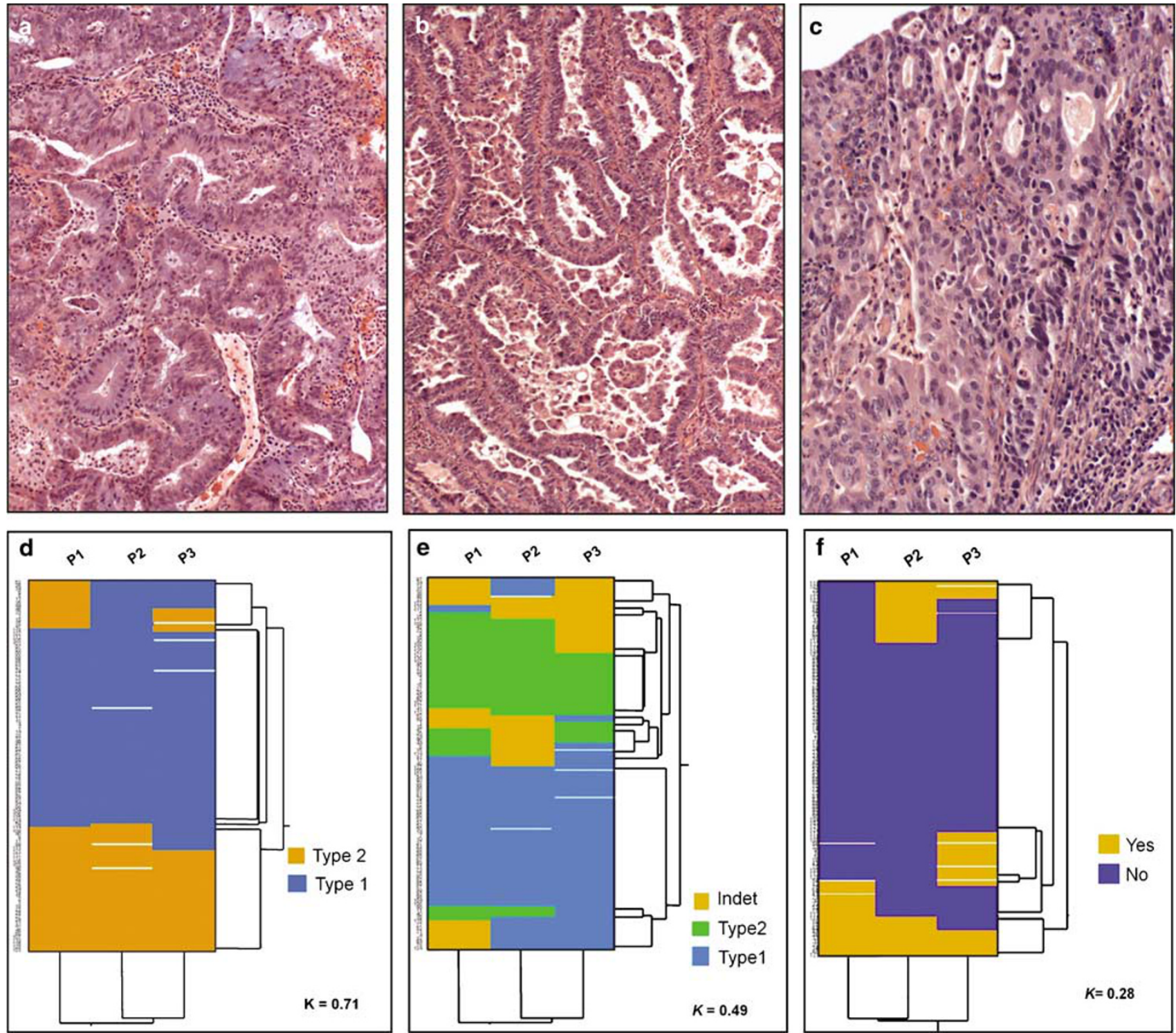

Figure 2 Examples of cases for which the reviewers achieved unanimous agreement of Type I (a) and Type II (b) carcinomas. Example of a case with non-unanimous agreement (c). Dendrograms depicting clustering of observer diagnoses (columns) by cases (rows) for a binary system (d) and three class system (e). Clustering of observer decisions regarding the need for p53 immunostaining is also depicted (f) (see Materials and methods).

\section{Discussion}

This study has demonstrated that a binary classification system of endometrial adenocarcinomas permits recognition of the two categories with excellent reproducibility. However, it also has shown that a minority of these tumors fall into a category that is not easily classified into either of the two, and that approximately one-third of these cases will pose problems for a majority (two of three) of observers. Indeterminate or intermediate histologic features, which resulted in assignment to the uncertain category included the following (Figure 3): (1) Mixed histotypes: some serous carcinomas are heterogeneous in their histologic appearance, being composed in part of patterns that are indistinguish- able from endometrioid differentiation. This group includes tumors that are homogeneously positive for p53 (ie serous carcinoma with endometrioid pattern) and tumors that are a bona fide hybrid of the two, in which p53-negative (endometrioid) and p53positive (serous) components co-exist. (2) Cytologicarchitectural discrepancy: some tumors with endometrioid architecture display an usually high degree of nuclear atypia and p53 immunopositivity. This is being increasingly recognized as a probable subset of serous carcinomas. ${ }^{6}$ (3) Similarity of villoglandular and papillary architecture: endometrioid tumors with a prominent villo-papillary architecture may superficially resemble the papillary pattern of Type II carcinomas, but are homogeneously p53negative. (4) Sample adequacy: accurate evaluation 

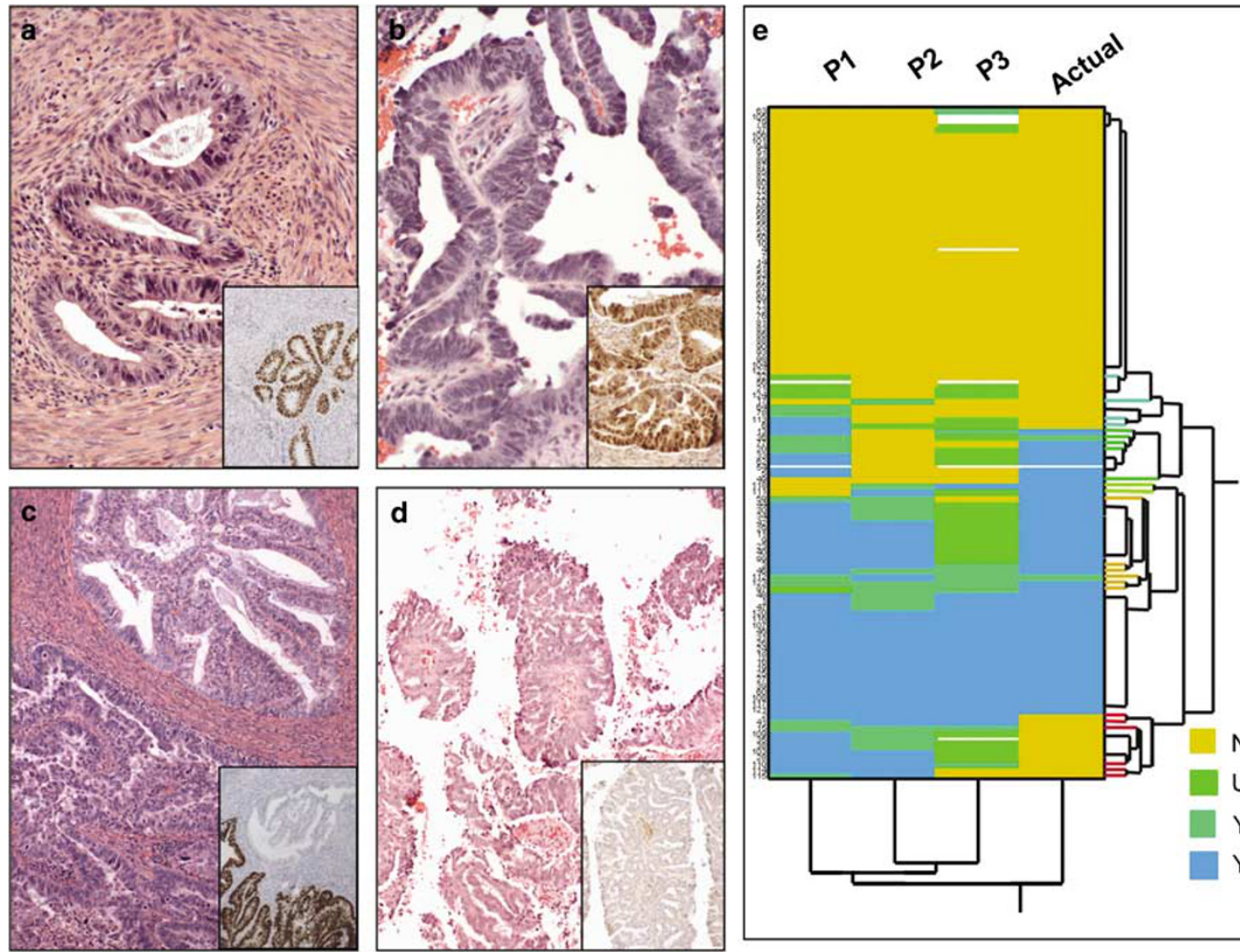

\section{No, Neg \\ Undecided \\ Yes, minor \\ Yes, diffuse}

$k=: 0.50$

Figure 3 Case examples with either non-unanimous agreement or re-classified as indeterminate by one or more observers, with p53 immunostaining. (a and b) Cases with endometrioid growth pattern and prominent nuclear atypia, both p53-positive (insets). (c) A biphasic pattern with both endometrioid (upper) and serous (lower) differentiation, the latter positive for p53 (inset). (d) Papillary architecture in a tumor, presumably endometrioid, p53 negative (inset). (e) A dendrogram compares staining predictions of the three observers with the actual staining interpretation (columns), for each case (rows).

Table 2 Diagnostic concordance and p53 outcomes by diagnostic schema

\begin{tabular}{|c|c|c|c|c|}
\hline Schema & Class & Agreement & N (\% of Type I/Type II) & P53-positive \\
\hline \multirow[t]{4}{*}{ Classic (2-class) } & Type I, $n=63$ & $2 / 3$ & $10 / 63(16 \%)$ & $7 / 10(70 \%)$ \\
\hline & & $3 / 3$ & $53 / 63(84 \%)$ & $8 / 53(15 \%)$ \\
\hline & Type II, $n=40$ & $2 / 3$ & $11 / 40(28 \%)$ & $8 / 11(73 \%)$ \\
\hline & & $3 / 3$ & $29 / 40(72 \%)$ & $25 / 29(86 \%)$ \\
\hline \multirow[t]{9}{*}{ Expanded (3-class) } & Type I, $n=65$ & $1 / 3$ & $14 / 65(22 \%)$ & $12 / 14(86 \%)$ \\
\hline & & $2 / 3$ & $13 / 65(20 \%)$ & $4 / 13(31 \%)$ \\
\hline & & $3 / 3$ & $38 / 65$ (58\%) & $1 / 38(3 \%)$ \\
\hline & Uncertain, $n=44$ & $1 / 3$ & $29 / 44(66 \%)$ & $16 / 30(53 \%)$ \\
\hline & & $2 / 3$ & $13 / 44(30 \%)$ & $11 / 13(85 \%)$ \\
\hline & & $3 / 3$ & $2 / 44(4 \%)$ & $2 / 2(100 \%)$ \\
\hline & Type II, $n=42$ & $1 / 3$ & $7 / 42(15 \%)$ & $4 / 7(57 \%)$ \\
\hline & & $2 / 3$ & $19 / 42(45 \%)$ & 15/19 (79\%) \\
\hline & & $3 / 3$ & $16 / 42(38 \%)$ & $14 / 16(88 \%)$ \\
\hline
\end{tabular}

may be compromised by fragmentation or scanty sampling making the case uninterpretable.

There is abundant literature supporting the poor prognosis of the serous (Type II) phenotype and the significance of p53 immunostaining as a helpful prognostic indicator. ${ }^{11}$ The relative roles of both nuclear atypia and p53 positivity as independent (of each other) prognostic markers is controversial, due in part to the fact that the two are so closely linked to serous carcinoma. ${ }^{12,13}$ However, investigators have verified the existence of p53-positive tumors with an endometrioid pattern, and in one study, p53 emerged as an important prognostic marker in this subset. $^{14,15}$ In view of the prognostic significance of p53 immunostaining with outcome, this marker has been proposed by some as a useful adjunct in the 
classification of selected endometrial adenocarcinomas.

Based on this study, a proportion of endometrial carcinomas can be expected to pose a problem in classification for a practicing pathologist, or be classified discordantly by two or more pathologists. The strength of association (or lack of) with p53, as shown in Table 2, increases as a function of agreement between observers, particularly in cases classified as endometrioid (Type I) and serous (Type II) carcinomas in a 3-tier system. Likewise, cases classified as indeterminate, particularly when two or more pathologists agree, carry a high rate of p53 positivity (Tables 2 and 3). Because the classification of a tumor as grade 2 or higher-either pre- or intraoperatively-might influence the decision to perform regional lymph node dissection, a high level of interobserver agreement is germane to consistent management. Whether p53 immunostaining of difficult cases will identify tumors at higher stage is less clear. ${ }^{16}$ Because the number of misclassifications is kept to a minimum when cases are reviewed by at least two observers, the case can be made for requiring review of endometrial malignancies by a second pathologist if the tumor cannot be readily classified as Type I. p53 immunostaining should be considered if the two observer diagnoses are not concordant.

\section{References}

1 Deligdisch L, Holinka C. Endometrial carcinoma: two diseases? Cancer Detect Prev 1987;10:237-246.

2 Mutter GL, Lin MC, Fitzgerald JT, et al. Altered PTEN expression as a diagnostic marker for the earliest endometrial precancers. J Natl Cancer Inst 2000;92: 924-930.

3 Hendrikson M, Ross J, Eifel P, et al. Uterine papillary serous adenocarcinoma: a highly malignant form of endometrial adenocarcinoma. Am J Surg Pathol 1982;6:93-108.

4 Sherman ME, Bur ME, Kurman RJ. p53 in endometrial cancer and its putative precursors: evidence for diverse pathways of tumorigenesis. Hum Pathol 1995;26: 1268-1274.
5 Wheeler DT, Bell KA, Kurman RJ, et al. Minimal uterine serous carcinoma: diagnosis and clinicopathologic correlation. Am J Surg Pathol 2000;24: 797-806.

6 Darvishian F, Hummer AJ, Thaler HT, et al. Serous endometrial cancers that mimic endometrioid adenocarcinomas: a clinicopathologic and immunohistochemical study of a group of problematic cases. Am J Surg Pathol 2004;28:1568-1578.

7 Boruta II DM, Gehrig PA, Groben PA, et al. Uterine serous and grade 3 endometrioid carcinomas: is there a survival difference? Cancer 2004;101:2214-2221.

8 Zheng W, Liang SX, Yu H, et al. Endometrial glandular dysplasia: a newly defined precursor lesion of uterine papillary serous carcinoma. Part I: morphologic features. Int J Surg Pathol 2004;12:207-223.

9 Ambros RA, Sherman ME, Zahn CM, et al. Endometrial intraepithelial carcinoma: a distinctive lesion specifically associated with tumors displaying serous differentiation. Hum Pathol 1995;26:1260-1267.

10 Alkushi A, Lim P, Coldman A, et al. Interpretation of p53 immunoreactivity in endometrial carcinoma: establishing a clinically relevant cut-off level. Int J Gynecol Pathol 2004;23:129-137.

11 Osmanagaoglu MA, Kadioglu S, Osmanagaoglu S, et al. The relationship between mutant p53 gene, DNA contents and conventional clinicopathological prognostic variables in cases with endometrial carcinoma. Eur J Gynaecol Oncol 2005;26:64-70.

12 Ozalp S, Yalcin OT, Tanir HM, et al. p53 overexpression as a prognostic indicator in endometrial carcinoma. Eur J Gynaecol Oncol 2003;24: 275-278.

13 Coronado PJ, Vidart JA, Lopez-asenjo JA, et al. P53 overexpression predicts endometrial carcinoma recurrence better than HER-2/neu overexpression. Eur J Obstet Gynecol Reprod Biol 2001;98:103-108.

14 Okuda T, Otsuka J, Sekizawa A, et al. p53 mutations and overexpression affect prognosis of ovarian endometrioid cancer but not clear cell cancer. Gynecol Oncol 2003;88:318-325.

15 Lundgren C, Auer G, Frankendal B, et al. Prognostic factors in surgical stage I endometrial carcinoma. Acta Oncol 2004;43:49-56.

16 Bonfitto VL, de Angelo Andrade LA. p53, estrogen and progesterone receptors in diagnostic curettage for endometrial adenocarcinoma and their correlation with morphological data and disease stage at hysterectomy. Sao Paulo Med J 2003;121:163-166. 